\title{
NOTE ON CARSON'S INTEGRAL EQUATION
}

\author{
BY J. J. SLADE, JR.
}

The theory of Heaviside's operational methods has been developed in various papers. Wiener* has perhaps given the most rigorous theory of these operators. Carson $\dagger$ has certainly given the most elaborate.

For the manipulation of these operators Carson's methods appear to be the most direct. The solution of the operational equation

$$
f(p)=\phi(t)
$$

is identified with the solution of the integral equation $\ddagger$

$$
f(p)=p \int_{0}^{\infty} \phi(t) e^{-p t} d t
$$

in which the real part of $p$ is positive, the path of integration is along the real axis, and $\phi(t)$ is to be defined for positive real values of $t$.

Dalzell§ has pointed out that the general solution of the equation is

$$
\phi(t)=\frac{1}{2 \pi i} \int_{c-i \infty}^{c+i \infty} \frac{f(p)}{p} e^{p t} d p,
$$

and March\| has shown that Bromwich's contour integrals satisfy it.

*The operational calculus, Mathematische Annalen, vol. 95 (1925), pp. 557-584.

† The Heaviside operational calculus, this Bulletin, vol. 32 (1926), pp. 4368; Electric Circuit Theory and the Operational Calculus, McGraw-Hill, 1926; Asymptotic solution of an operational equation, Transactions of this Society, vol. 31 (1929), pp. 782-792.

$\ddagger$ Carson, this Bulletin, loc. cit.

$\S$ Heaviside's operational method, Proceedings Physical Society, vol. 42 (1930), pp. 75-81.

|| The Heaviside operational calculus, this Bulletin, vol. 33 (1927), pp. 311-318. For Bromwich's theory, see Jeffreys, Operational Methods in Mathematical Physics, Cambridge Tract No. 23, 1931, Chapter 2. 
Carson* has noticed the relation between equation (1) and Borel's integral summation formula, and he has suggested that Borel's summation methods might be employed in obtaining the asymptotic expansion of $\phi(t)$ when $f(p)$ is obtained in increasing fractional powers of $p$.

In the present paper this investigation is carried through, and it is found that Borel's theory of the summation of divergent series furnishes a theory for equation (1) which at once is direct and throws light on the nature of the series solutions of it.

We may assume at first that $\infty$ is a regular point of $f(p)$ as a function of $p^{\lambda},(0 \leqq \lambda \leqq 1), \dagger$ so that, for sufficiently large values of $p$, we may write

Thenf

$$
f(p)=\sum_{r} a_{r}\left(p^{\lambda}\right)^{-r}
$$

where

$$
f(p)=\int_{0}^{\infty} u(\xi) e^{-\xi} d \xi
$$

$$
u(\xi)=\sum_{r} a_{r}\left(p^{\lambda}\right)^{-r} \frac{\xi^{r}}{r !} .
$$

If in (1) we place $p t=\xi$, then Carson's equation is transformed into Borel's integral relation, where

$$
u(\xi)=\phi\left(\frac{\xi}{p}\right)=\sum_{r} a_{r}\left(p^{\lambda}\right)^{-r} \frac{\xi^{r}}{r !} .
$$

Now if we can transform the right hand member of (3) so that $p$ does not appear in the expansion of $\phi$, then (3) will be the solution of (1) in terms of the expansion of $f(p) . \S$ To do this

* Loc. cit., this Bulletin, p. 49 (note), and p. 68.

$\dagger$ Heaviside's method has been applied to the solution of equations of the type $\left(\nabla^{2}-\mu\right) \phi=0$, where $\mu=a+b(\partial / \partial t)+c\left(\partial^{2} / \partial t^{2}\right)$. From such a source $\mu$ is either 1 or $1 / 2$. However, the theory may be extended to apply to the solution of $(D-\mu) \phi=0$, where $D$ is a general operator in $x, y, \cdots$ and $\mu$ is a linear operator in $t$, and thus $\lambda$ may take on other values. But regardless of the form $f(p)$ may take as a functional operator, equation (1), considered merely as an integral equation, makes sense for any $\lambda$.

$\ddagger$ See, for example, Ford, Studies on Divergent Series and Summability, Michigan Science Series, II, Macmillan, 1916, p. 78 et seq.

$\S$ It will still be a solution when $p$ appears in the expansion, but it will not be explicit, for, in that case, $p$ must be interpreted as an operator. See equation (8) below. 
place $\xi=p \xi$ in the integral

Then

$$
\Gamma(r \lambda+1)=\int_{0}^{\infty} \xi^{r \lambda} e^{-\xi} d \xi .
$$

and

$$
\left(p^{\lambda}\right)^{-r}=p \int_{0}^{\infty} \frac{\zeta^{r \lambda}}{\Gamma(r \lambda+1)} e^{-p \zeta} d \zeta,
$$

$$
\phi\left(\frac{\xi}{p}\right)=p \sum_{r} a_{r} \frac{\xi^{r}}{r !} \int_{0}^{\infty} \frac{\zeta^{r \lambda}}{\Gamma(r \lambda+1)} e^{-p \zeta} d \zeta .
$$

Since by hypothesis $f(p)$ is absolutely convergent (in the neighborhood of $\infty$ ), so will the series for $\phi$ be in some interval, and in equation (5), $\sum \int=\int \sum$. We may then write

and hence

$$
\begin{aligned}
f(p) & =p \int_{0}^{\infty} \int_{0}^{\infty}\left\{\sum_{r} a \frac{\xi^{r}}{r ! \Gamma(r \lambda+1)}\right\} \xi^{r \lambda} e^{-p \zeta} e^{-\xi} d \zeta d \xi \\
& =p \int_{0}^{\infty}\left\{\sum_{r} a_{r} \frac{\zeta^{r \lambda}}{\Gamma(r \lambda+1)}\right\} e^{-p \zeta} d \zeta,
\end{aligned}
$$

$$
\phi(t)=\sum_{r} a_{r} \frac{t^{r \lambda}}{\Gamma(r \lambda+1)},
$$

which gives the well known transformation for the series (2):

$$
p^{-r \lambda}=\frac{t^{r \lambda}}{\Gamma(r \lambda+1)} .
$$

Suppose now that $f(p)$, as a function of $p^{\lambda}$, is regular at $p=0$, and that its singularities lie to the left of the imaginary axis. Then for sufficiently small values of $p$,

$$
f(p)=\sum_{r} a_{r}\left(p^{\lambda}\right)^{r}
$$

The integrals (4) are not defined for positive exponents of $p$ after a sufficiently large $r$. In this case replace $p^{r \lambda}$ in the series corresponding to (3) by the right hand member of the identity

$$
p^{r \lambda}=\frac{p^{k+1}}{\prod_{j=1}^{k}(j-r \lambda) \Gamma(1-r \lambda)} \int_{0}^{\infty} \zeta^{k-r \lambda} e^{-p \zeta} d \zeta,
$$


in which $k$ is a positive integer such that $k<r \lambda<k+1$. Proceeding as before we get, instead of (6),

$$
p^{r \lambda}=\frac{p^{k} t^{k-r \lambda}}{\prod_{j=1}^{k}(j-r \lambda) \Gamma(1-r \lambda)} .
$$

To transform the right hand member of (8) so that $p$ does not appear we first introduce a set of functions $p_{k}$ which satisfy the following requirements: $p_{0}$ is monotonic increasing from 0 to $\infty$, and

$$
\left\{\begin{array}{l}
p_{0}=0, t=0 ; p_{0}=1, t=\infty ; p_{0} \rightarrow 1 \text { as } t \text { approaches } \delta>0 \\
\text { from the left, } \delta \text { being an arbitrarily small number; } \\
\int_{0}^{t} p_{k} d t=p_{k-1}, \\
\lim _{\substack{t \rightarrow 0 \\
n \rightarrow \infty}} t^{-n} p_{k}=0, \lim _{\substack{t \rightarrow \infty \\
n \rightarrow \infty}} t^{n} p_{k}=0, \quad(k \neq 0 \text { in this last }) .
\end{array}\right.
$$

An analytic function which satisfies all the requirements for $p_{0}$ is

$$
\frac{1}{\pi^{1 / 2}} \int_{-\infty}^{h \log (2 t / \delta)} e^{-\xi^{2}} d \xi
$$

where $h$ is an arbitrarily large positive real number.*

In the first place it is seen that

$$
p^{k}=p_{k} .
$$

For, consider the integral

$$
p \int_{0}^{\infty} p_{k} e^{-p t} d t
$$

* The function $p_{0}$ here used is a modification of Heaviside's unit function $1 \equiv \frac{1}{2}(1+\operatorname{sgn} t)$. For a discussion of this function see Bush, Operational Circuit Analysis, Wiley, 1929, p. 41 . The physical meaning of the "impulse" $p_{1}$ (there called $p 1$ ) is discussed on p. 122 . On p. 258 et seq. are introduced implicitly a set of functions similar to the $p_{k}$, except that generally $\lim _{t \rightarrow 0} t^{-n} p_{k} \neq 0$ as defined there. The function $p_{0}$, as defined here, meets more closely than does $\frac{1}{2}(1+\operatorname{sgn} t)$ the requirements of physical theory. 
Integrating by parts, we find

$$
-\left.p e^{-p t} p_{k-1}\right|_{0} ^{\infty}+p^{2} \int_{0}^{\infty} p_{k-1} e^{-p t} d t=p^{k+1} \int_{0}^{\infty} p_{0} e^{-p t} d t=p^{k},
$$

since

$$
\int_{0}^{\infty} p_{0} e^{-p t} d t \rightarrow \int_{0}^{\infty} e^{-p t} d t, \text { as } \delta \rightarrow 0 .
$$

This establishes (10). Now let

$$
\prod_{j=1}^{k}(j-r \lambda) \Gamma(1-r \lambda) \Psi_{0}(r \lambda, t)=t^{k-r \lambda} p_{0},
$$

where $k$ has the same meaning as in (7). Also let

Consider the integral

$$
\frac{\partial^{n} \Psi_{0}}{\partial t^{n}}=\Psi_{n}
$$

$$
p \int_{0}^{\infty} \Psi_{k} e^{-p t} d t=-\left.p e^{-p t} \Psi_{k-1}\right|_{0} ^{\infty}+p^{2} \int_{0}^{\infty} \Psi_{k-1} e^{-p t} d t .
$$

Because of the relations (9) the integrated part vanishes at both limits. Proceeding in this way, we get

$$
\begin{aligned}
& p \int_{0}^{\infty} \Psi_{k} e^{-p t} d t=p^{k+1} \int_{0}^{\infty} \Psi_{0} e^{-p t} d t \\
&=\frac{p^{k+1}}{\prod_{j=1}^{k}(j-r \lambda) \Gamma(1-r \lambda)} \int_{0}^{\infty} t^{k-r \lambda} p_{0} e^{-p t} d t \\
& \rightarrow \frac{p^{k+1}}{\prod_{j=1}^{k}(j-r \lambda) \Gamma(1-r \lambda)} \int_{0}^{\infty} t^{k-r \lambda} e^{-p t} d t, \text { as } \delta \rightarrow 0 .
\end{aligned}
$$

Finally, (8) becomes

(11) $p^{r \lambda}=\Psi_{k}(r \lambda, t)=\frac{t^{-r \lambda}}{\Gamma(1-r \lambda)}\left[p_{0}+\left(\begin{array}{l}k \\ 1\end{array}\right) \frac{t p_{1}}{(1-r \lambda)}\right.$

$$
\left.+\left(\begin{array}{l}
k \\
2
\end{array}\right) \frac{t^{2} p_{2}}{(1-r \lambda)(2-r \lambda)}+\cdots+\frac{t^{k} p_{k}}{\prod_{j=1}^{k}(\jmath-r \lambda)}\right] \text {. }
$$


The possibility of setting $p_{k}=0, k>0$, for large values of $t$ will depend on whether $\phi(t)$ admits an asymptotic expansion along the positive real axis. The region of definition of $\phi(t)$ as well as the possibility of its expansion by the transformation (11) may be inferred from general theorems in the theory of divergent series*. With due regard to the distribution of the singularities of $f(p),(6)$ and (11) may be used when $f(p)$ is expanded in a two-way Laurent series.

RUTGERS UNIVERSITY

\section{SYMMETRIC, SELF-DUAL, RATIONAL PLANE CURVES OF ODD ORDER $\dagger$}

BY D. C. DUNCAN

The writer has established the existence $\$$ of the real, nondegenerate, completely symmetric, self-dual, elliptic curves of order $4 k+2$, and has indicated an extremely simple plan of sketching the loci approximately. In pursuing the program there indicated, we now establish the existence of completely symmetric rational self-dual curves of order $2 k+1$, and indicate the corresponding easy mode of sketching them approximately. These loci also are invariant under $4 k+2$ collineations and $4 k+2$ correlations of which $2 k+1$ are polarities by real rectangular hyperbolas and one a polarity by an imaginary circle. Moreover, the $(2 k+1)^{2}$ foci have an interesting distribution and admit rather easy determination. In conclusion, the equations in Cartesian coordinates of the curves of the lowest three orders, 5, 7, and 9, are listed, together with a sketch of the 9-ic depicting all singular elements, real foci, and real autopolarizing conics. In a subsequent paper the elliptic and rational self-dual completely symmetric curves of orders $4 k, 2 k+1$, and $4 k, 4 k+2$, respectively, are to be exhibited, therewith terminating the present discussion.

* See, for example, Borel et Bouligand, Leçons sur les Séries Divergents, Gauthier-Villars, 1928, Chapter 4.

$\dagger$ Presented to the Society under a slightly different title, December 2, 1933.

$\ddagger$ This Bulletin, vol. 39 (1933), pp. 809-813 